\title{
A Study on Prevalence and Causative Factors of Megaloblastic Anaemia in Hadoti Region
}

\author{
Lakshmi agarwal $^{1 *}$, Jayant Ramawat ${ }^{1}$, Manmohan Agrawala ${ }^{2}$ and Naresh Rai ${ }^{1}$ \\ ${ }^{1}$ Govt Medical College, Kota, Rajasthan, India \\ ${ }^{2}$ Cosultant Oncosurgeon, Pushpadi Cancer Care Centre, Kota, Rajasthan, India
}

\begin{abstract}
Background: Anaemia is the most common problems encountered by clinicians in Hadoti Region. The affected population includes male, female as well as children. Over the last two decades, it has been found that incidence of megaloblastic anaemia is increasing. Folic Acid and Vitamin B12 deficiency are the most common cause of megaloblastic anemia. Of these two micronutrients, Vitamin B12 deficiency is more common now, due to vegetarian life style of people. At present, Anemia control or prophylaxis program give only Iron and Folic acid. This study has been chosen to focus on this issue. The cases for increasing incidences of Folate / Vit. B12 deficiency needs to be elucidated.

Objectives: To focus on the incidence of megaloblastic anaemia in Hadoti Region. \& Probable Causative factors will be analysed

Methods: All patients presenting to our hospital over a period of 2 months with a haemoglobin $<10 \mathrm{~g} / \mathrm{dl}$ and peripheral smear findings consistent with megaloblastic anaemia will be included in the study. Diet, drug intake, previous blood transfusion, presenting symptoms and other relevant history will be taken into consideration. Complete blood counts, peripheral film examination, reticulocyte count and cobalamin and folate assays will be recorded. Patients with chronic disease like renal disease, cancer, tuberculosis, liver disease etc will be excluded from the study. All data will be collected and evaluated statistically.

Result: In the present study, total 500 patients who were admitted in medicine, paediatric and gynaecology ward were evaluated. All these patients met the inclusion criteria. Depending on the MCV value, serum assay and peripheral smear finding, they were categorised into 3 groups- Macrocytic, normocytic and microcytic anaemia. The normal MCV value but with megaloblastic blood film or low serum markers were considered into macrocytic anaemia. Total 100 patients were diagnosed as macrocytic anaemia. The sex distribution were-70(male), 30 (female). Fifty five per cent of patients with cobalamin deficiency and $08 \%$ of patients with folate deficiency were found. All the patients were vegetarian and from middle class and low socio economic group.

Conclusion: Cobalamin deficiency was responsible for megaloblastic anaemia in the majority of our patients. The supplementation program for Anemia control and prophylaxis should vary according to the regional requirements. Vitamin B12 should be included in the nutritional programme along with iron and folic acid. Awareness camp and Education program about megaloblastic anemia can be implemented for the prevention.
\end{abstract}

Keywords: Megaloblastic Anemia, Increasing, Vitamin B12, Health Programme

\section{Introduction}

Anaemia is the most common problems encountered by clinicians in Hadoti Region. The affected population includes male, female as well as children. According to World Health Organization (WHO), the global prevalence of anaemia is $24.8 \%$, which means about 1.62 billion people worldwide ${ }^{1}$.Overall health status of a person is judged on level of haemoglobin of a person. The high prevalence of anaemia is a serious health hazards for the economic development and productivity of the country

Over the last two decades, it has been found that incidence of megaloblastic anaemia is increasing. Folic Acid and Vitamin B12 deficiency are the most common cause of megaloblastic anaemia. Of these two micronutrients, Vitamin B12 deficiency is more common now, due to vegetarian life style of people. Vegetarianism has become increasingly popular among people including adolescents in current years perhaps because this diet is believed to offer health benefits.

Cobalamin is synthesized by bacteria and is found in soil and in contaminated water. Foods of animal origin(e.g.meat,eggs,and milk) are the primary dietary sources. The amount of cobalamin in the average Western diet ( 5 to $15 \mathrm{mg} /$ day) is more than sufficient to meet normal requirements. The body can store large amounts of cobalamin. Because of this, it can take 2 to 5 years for adeficiency to develop even in the presence of severe malabsorption ${ }^{2}$.

As the totally vegetarians do not consume any animal products, they are at high risk of developing vitamin B12 
deficiency. It is generally agreed that in some communities the only source of vitamin B12 is from contamination of food by microorganisms. When vegetarians move to countries where there are high standards of hygiene and the rules of sanitation are stringent, there is good evidence that risk of vitamin B12 deficiency increases in adults and particularly in children born to and breastfed by women who are strict vegans ${ }^{3}$.

In India with diverse ethnic populations, different dietary and social customs, the incidence of megaloblastic anaemia and its associated problems have not been adequately documented. Unfortunately, from public health view point, deficiency of B12 / folate has been regarded to contribute little to nutritional anemia ${ }^{4}$. Various workers have brought this to the forefront but in vain. At present, anaemia control or prophylaxis program give only Iron and Folic acid. This study has been chosen to focus on this issue

\section{Material and Methods}

It is a prospective and retrospective study. All patients presenting to our hospital over a period of 2 months from 1staugust 2016 to $30^{\text {th }}$ September 2016 with a haemoglobin $<10 \mathrm{~g} / \mathrm{dl}$ and peripheral smear findings consistent with anaemia were included in the study. Diet, drug intake, previous blood transfusion, presenting symptoms and other relevant history were taken into consideration. Complete blood counts, peripheral film examination, reticulocyte count and cobalamin and folate assays were recorded. Patients with chronic disease like renal disease, cancer, tuberculosis; liver disease etc was excluded from the study.

\section{Results}

In the present study, total 500 patients who were admitted in medicine, paediatric and gynaecology ward were evaluated. All these patients met the inclusion criteria. Depending on the MCV value, serum assay and peripheral smear finding, they were categorised into 3 groups-Macrocytic, normocytic and microcytic anaemia (table-1). The normal MCV value but with megaloblastic blood film or low serum markers were considered into macrocytic anaemia. Total 100 patients were diagnosed as macrocytic anaemia. The sex distribution were-70(male), 30(female). Fifty five per cent of patients with cobalamin deficiency and $08 \%$ of patients with folate deficiency were found(table-2). All the patients were vegetarian and from middle class and low socio economic group.

Presentation: The predominant symptoms were

- Neurological symptoms (50\%),

- $\quad$ Fatigue (38\%)

- Anorexia,

- Gastritis,
- Low grade fever,

- Palpitation.

Laboratory findings: TheMCV ranged from $70 \mathrm{fL}$ to $128 \mathrm{fL}$.

Peripheral smear revealed Macro-ovalocytes, Tear drop cells, Basophilic stippling, Polychromasia, Hyper segmented neutrophils or Pancytopenia. 30 patients had received blood transfusions for anaemia

Table 1: distribution of different types of anaemia.

\begin{tabular}{|c|c|}
\hline Microcytic & 350 \\
\hline Normocytic & 50 \\
\hline Macrocytic & 100 \\
\hline Total & $\mathbf{5 0 0}$ \\
\hline
\end{tabular}

Table 2:- Distribution of megaloblastic anaemia according to the causative factors

\begin{tabular}{|c|c|}
\hline Pure cobalamin deficiency & $55 \%$ \\
\hline Pure folate deficiency & $10 \%$ \\
\hline Combined deficiency & $8 \%$ \\
\hline Unknown(reports not available) & $27 \%$ \\
\hline
\end{tabular}

\section{Discussion}

Megaloblastic anaemiais a heterogeneous group of disorders that have common blood abnormalities and symptoms. It is a macrocytic anaemia that is usually accompanied by leukopenia and thrombocytopenia and specific bone marrow morphology affecting erythroid, myeloid and platelet precursors. ${ }^{5}$. Megaloblastic anaemia most commonly results from folate or cobalamin (vitamin B12) deficiency.

Paul Ehrlich in 1880 first used the term megaloblast to describe the abnormal cells in the bone marrow of a patient with pernicious anaemia. Thomas Addison first described pernicious anaemia, the best known of the megaloblasticanaemia, in 1855 . For a number of years the disease was known as Addisoniananaemia ${ }^{6}$. Anton Biermer in 1872 first used the term, pernicious anaemia. Vitamin B12 was first identified in the 1920's by Minot and Murphy as being the extrinsic factor present in the liver which reverses the symptoms of pernicious anaemia, as reviewed by Markle and Okuda. The substance was isolated and named vitamin B12 in 1948. Hodgkin and coworkers determined the crystalline structure of vitamin B12 molecule in the $1950^{7}$. Vitamin B12 is a term used for a group of physiologically active substances, which are chemically classified as cobalamin. The basic structure is a tetrapyrrole ring, acorrin, with a central cobalt atom and a purine nucleotide (5, 6-dimethylbenzimidazole) linked to it. 
The incidence of megaloblastic anaemiais increasing, althoughevidence for this may not be forthcoming easily. The diagnosis of vitamin B12 deficiency is not straightforward. There are no uniform diagnostic criteria and no single laboratory test constitutes a gold standard. Various criteria have been used in the literature for the diagnosis of this anaemia which includes macrocytosis of red cells, presence of megaloblastic changes in thebone marrow and subnormalmicronutrient levels. Gera et al shows an almost four fold rise in proportion of macrocytic anaemia cases over less than a decade at one center-2 \% in 1991 and $7.8 \%$ in $1999^{\circ}$. A recent report reveals that $46.9 \%$ of non-anaemic adult subjects having subnormal levels of B12 or folate deficiency- vitamin B12 being five times more common than that of folate.A study from Mexico showed B12 deficiency in 19\% - 41\% of various population subgroups while no cases with folate deficiency were observed ${ }^{9}$. Another study on Guatemalan lactating mothers revealed B12 deficiency in $46 \%$ compared to $9 \%$ prevalence of folate deficiency ${ }^{10}$. Series on megaloblastic anaemia patients from Pakistan and Zimbabwe revealed B12 deficiency inover $50 \%$ cases while folate deficiency was seen in only $8 \%$ and $17 \%$ cases, respectively ${ }^{11}$.

Sarode et al ${ }^{12}$ from Chandigarh reported B12 deficiency in nearly $85 \%$ cases of megaloblastic anemia. The same finding was also observed by Mukibi et al ${ }^{13}$, Khanduri et al ${ }^{14,16}$ and various other workers. Gomber $\mathrm{S}$ et al in $1998^{15}$ conducted a study on Prevalence \&aetiology of nutritional anaemia's in early childhood in an urban slum. Randomly selected 300 children aged 3 months- 3 year were analysed over a period of one year for estimating prevalence of nutritional anaemia. Pure iron deficiency anaemia (IDA) was detected in $41.4 \%$ of anaemic children. Vitamin B12 deficiency alone or in combination with iron was diagnosed in 14.4 and 22.2 per cent anaemic children respectively

In the present study, microcytic anaemia was found to be the most common cause of anaemia in Hadoti region followed by megaloblastic anaemia.Cobalamin deficiency was responsible for megaloblastic anaemia in the majority of our patients $(55 \%$ pure cobalamin deficiency and $10 \%$ combined deficiency) and pure folate deficiency in $8 \%$. The serum assay of vitamin was not available in $27 \%$ of the patients. The MCV was found to be $>90 \mathrm{fl}$ in $80 \%$ of the cases. In the rest of the cases, though MCV was normal, peripheral smear findings were characteristic. It includes Macro-ovalocytes, hyper segmented neutrophils, basophilic stippling. This could be because of concomitant iron deficiency.Pancytopenia was found in a few patients.

Complete blood count, peripheral smear and the serum assay of vitamins are the diagnostic tests of megaloblastic anaemia. Bone marrow can also be done for the diagnosis. Since megaloblastic anaemia is a chronic disorder, most of the patients are well compensated. The symptoms arises when the haemoglobin is very low $<4 \mathrm{gm} \%$. Blood transfusion is not urgency in such cases. The folate and vitamin B12 deficiency should be differentiated by the serum assay before the therapy is started.

Most of the patients were found to be vegetarian.Even those who consider themselves as non-vegetarians usually consume meat only occasionally.Megaloblastic anaemia seen in infants and young children may be attributed to maternal deficiency which results in poor body stores at the time of birth.Cobalamincontent of breast milk is lower in vegetarian mothers and is directly proportional to serum cobalaminlevels. Brit et al alsoobserved higher prevalence of megaloblastic anaemia among vegetarian Indian settlers in $\mathrm{UK}^{17}$. The above findings suggest that most cases of megaloblastic anaemia are caused by nutritional deficiency of vitamin B12, folate or both. The other causes of deficiency account for minority of cases.

Irrespective of the cause, vitamin B12 deficiency now appears to be more common than folatedeficiency in causing nutritional macrocytic anaemia.In India, the national nutritional program for anaemia control or prophylaxis gives only Iron and Folic acid. The various studies done earlier and the present study highlight the rise in incidences of vitamin B12 deficiency which needs to be elucidated. The government should acknowledge the burden of vitamin B12 deficiency and implement it in the anaemia control program. The vitamin B12 tablet should be distributed along with iron and folic acid. The other option which can be considered is fortifying foods with vitamin B12. This can help in achieving the target of decreasing the incidence of vitamin B12 deficiency and reduction of subclinical vitamin B12 deficiency. A few workers have suggested that fortification of foods with vitamin B12 may not be as effective due to malabsoption. But, in India inadequate diet is the main cause rather than absorption.

\section{Conclusion}

The incidences of megaloblastic anaemia come next to iron deficiency anaemia. Data regarding the magnitude of the problem in Hadoti region of Rajasthan and the factors that might influence its incidence are lacking. Vitamin B12 deficiency causing megaloblastic anaemia is associated with other systemic manifestation and the morbidity is more. Our anaemia control programme only takes care of iron and folic acid deficiency. Vitamin B12 should be included in the national nutritional programme especially in the region where majority of the people are vegetarian. The Physician must keep in mind vitamin B12 deficiency in their differential diagnosis of anaemia. 


\section{References}

1. Sachdev HPS, Chandhary P. Nutrition in children: Developing country concerns Reprint. 1995; 1

2. Baker SJ, DeMaeyer EM. Nutritional anemia; its understanding and control with special reference to work of World Health Organization. Amer J ClinNutr 1979; 32: 368-417

3. Specker BL, Black A, Allen L et al. Vitamin B: Low milk concentration are related to low serum concentrations in vegetarian women and methylmalonicaciduria in their infants. Amer J ClinNutr 1990; 52: 1073-1076.

4. Chandra J. Megaloblastic anemia: Back in focus. Indian J Pediatr 2010; 77:795-9.

5. Antony AC. Megaloblastic anemia. In: Hoffman R, Benz EJ, Shattil SJ, FurieB,Cohen HJ, Silberstein LE, et al. (eds). Hematology. Basic principles and practice.4th ed. Edinburgh:Churchill Livingstone; 2005:519-56.

6. Addison T. Anaemia-disease of the suprarenal capsules. London Med Gazette1849;43:517-18.

7. GRichardLee, JohnFoerster, John Lukens, FrixosParaskevas, John P Greer, George M Rodgers, Wintrobe's Clinical Hematology 10th edition 1998, Williams \&Wilkins A Waverly company volume 1 page 942 .

8. Gera R, Singh ZN, Chaudhury P. Profile of nutritional anemia in hospitalized children over a decade. Conference Abstracts,38thNational conference of Indian academy of Pediatrics Patna 2001; HO-09, pp 60
9. Allen LH, Rosado JL, Casterline JE et al. Vitamin B12 deficiency and malabsorption are highly prevalent in Mexican communities. Amer J ClinNutr 1995; 65: 1013-1019.

10. Casterline JE, Allen LH, Ruel MT. Vitamin B12 deficiency is very prevalent in lactating Guatemalan women and their infants at three months postpartum. J Nutr 1997; 127: 1966-1970

11. Madood-ul-Mannan, Anwar M, Saleem M et al. Study of serum vitamin B12 and folate levels in patients of megaloblastic anemia in northern Pakistan. J Pak Med Assoc 1995; 45: 187-188.

12. Sarode R, Garewal G, Marwaha N, Marwaha RK, Varma S, Ghosh K, Pancytopenia in nutritional megaloblastic anemia: A study from north-west India. Trop Geogr Med 1989; 41:331-6.

13. Mukibi JM, Makumbi FA, Gwanzura C. Megaloblastic anemia in Zimbabwe: Spectrum of clinical and haematological manifestations. East Afr Med J 1992; 9: 8387.

14. Khanduri U, Sharma A, Joshi A. Occult cobalamin and folate deficiency in Indians. Natl Med J India 2005;18:182-3.

15. GomberS,KumarS,RusiaU,GuptaP,AgarwalKN,SharmaS. Prevalence and etiology nutritional anaemia in early childhood in an urban slum Indian J med Res.1998;73:107-269.

16. Khanduri U, Sharma A. Megaloblastic anaemia: Prevalence and causative factors. Natl Med J India 2007;20:172-5

17. Britt RP, Harper C, Spray GH. Pernicious anaemia in Indian immigrants in London area. Br J Haematol 1970; 18: 637-642.

*Corresponding author:

Dr Lakshmi Agarwal, 34 A, Shreenathpuram, Near Ahinsha Circle, Kota, Rajasthan, India

Phone: +919772464587

Email: drlaxmiagarwal@gmail.com

Acknowledgement : STS ICMR

Date of Submission : 05.02.2017

Date of Acceptance : 13.02.2017

Financial or other Competing Interests: None.

Date of Publication : 21.02.2017

http://www.pacificejournals.com/aabs 